\title{
A Well-Oiled Stereodynamic Probe
}

Key words

axial chirality

stereodynamic probe

chiroptical response

induced circular dichroism

$\mathrm{Pd}\left(\mathrm{PPh}_{3}\right)_{4}(10 \mathrm{~mol} \%)$ Cul (10 mol\%)

$88 \%$ yield
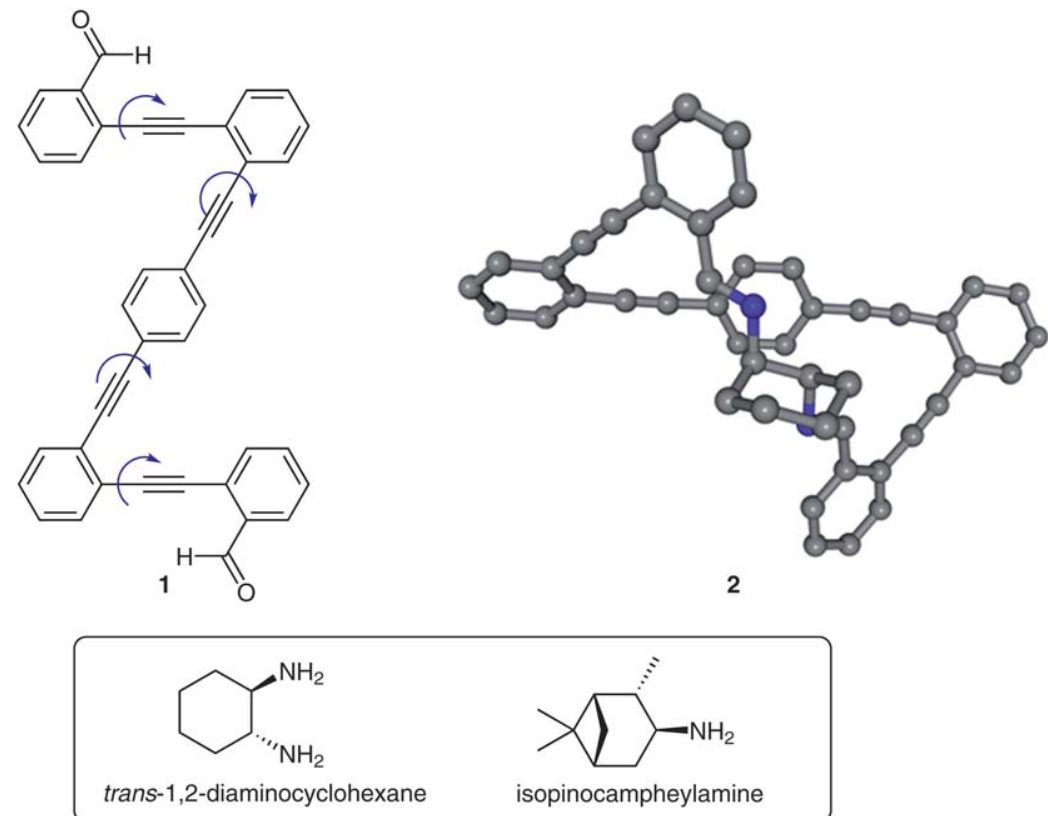

Significance: A series of Sonogashira crosscoupling reactions is employed in the synthesis of the aryl-acetylene stereodynamic probe $\mathbf{1}$, which was obtained in $62 \%$ overall yield. While $\mathbf{1}$ is achiral, condensation with chiral di- or monoamines produces axially chiral diimines (e.g. 2). Because of the unhindered rotation in $\mathbf{1}$ (blue arrows) the central-to-axial chirality induction is essentially 'frictionless'.

SYNFACTS Contributors: Timothy M. Swager, Olesya Haze Synfacts 2011, 5, 0493-0493 Published online: 15.04.2011 Dol: 10.1055/s-0030-1259795; Reg-No.: S02811SF
Comment: Extraordinary chirality amplification and strong Cotton effects were observed, and were used for in situ induced circular dichroism (ICD) analysis of a variety of chiral amines. CD amplitudes showed linear dependency on \% ee, and enantiomeric excess was determined within $4 \%$ of the actual values for trans-1,2-diaminocyclohexane, and within $5.5 \%$ for the isopinocampheylamine. 